\title{
Estimation of the Channel Impulse Response for GSM System
}

\author{
Jacek Stefański \\ Technical University of Gdansk, Department of Radiocommunication \\ e-mail: jstef@eti.pg.gda.pl
}

Keywords: GSM, MLSE, training sequence.

\begin{abstract}
The aim of this article is to present an improved training sequence for estimating the non-stationary channel impulse response used in GSM.Many components of the received signal in digital communication systems arrive to the receiver antenna with different delays. These delayed signal components can cause intersymbol interference, increase BER (Bit Error Rate) and hence degrade quality of the received source information. Therefore, in order to increase the quality of received signal, every transmitted data packet contains a training sequence. However, the training sequence recommended by GSM is not the optimal method of estimating the channel impulse response. This can be clearly portrayed in a simulation performance.
\end{abstract}

\section{INTRODUCTION}

In digital cellular mobile communication systems, such as GSM (Global System for Mobile Communications), intersymbol interference which occurs due to a time-variant multipath fading must be neutralized by the application of adaptive equalizers.

A MLSE (Maximum Likelihood Sequence Estimator) represents the optimal receiver structure [1]. The MLSE receiver consists of matched filter and a Viterbi processor. The received signal is sampled and each sample is filtered through a matched filter whose parameters are approximated by 
training sequence. Viterbi processor equalizers estimate transmitting symbol sequence by using the Viterbi algorithm.

The number of matched filter taps depends on the maximal echo delays which in turn determine the number of states in the Viterbi processor (the number of matched filter taps for GSM is five).

\section{GMSK MODULATION}

A GMSK (Gaussian Minimum Shift Keying) [2] modulated signal can be represented as

$$
s(t)=e^{j \theta(t)}, \theta(t)=\theta_{0}+\sum_{i} b_{i} \phi\left(t-i T_{b}\right)
$$

where $\theta_{0}$ is an initial phase, $T_{b}$ is the bit period and $b_{i} \in\{1,-1\}$ are the differentially encoded data bits. In terms of the raw data bits $a_{i} \in\{0,1\}$, $b_{i}=1-2\left(a_{i} \oplus a_{i-1}\right)$, where $\oplus$ denotes modulo 2 addition [3]. The phase pulse-shaping function $\phi(t)$ is given by

$$
\phi(t)=K \cdot \int_{-1,5 T_{b}}^{t} y(\tau) d \tau
$$

(2)

$$
\begin{aligned}
& \text { where: } y(t)=\frac{A}{\sqrt{2 \pi}}\left\{Q\left[\frac{2 \pi B T_{b}}{\sqrt{\ln 2}}\left(\frac{t}{T_{b}}-\frac{1}{2}\right)\right]-Q\left[\frac{2 \pi B T_{b}}{\sqrt{\ln 2}}\left(\frac{t}{T_{b}}+\frac{1}{2}\right)\right]\right\} \text {, } \\
& Q(x)=\frac{1}{\sqrt{2 \pi}} \int_{x}^{\infty} e^{-\frac{t^{2}}{2}} d t
\end{aligned}
$$

$B T_{b}=0.3$ (for GSM),

$A, K$ - constans.

To implement the Viterbi algorithm, it is imperative to determine a finite number of states for the GMSK modulated signal. This can be accomplished by modifying the phase pulse-shaping function $\phi(t)$ from fig. $1 . \phi(t)$ is approximated as [4]. 


$$
\hat{\phi}(t)=\left\{\begin{array}{llr}
0 & \text { for } & t<-T_{b} \\
\phi(t)+\phi\left(-1,5 T_{b}\right)-\phi\left(-1,5 T_{b}-t\right) & \text { for } & -T_{b}<t<-\frac{T_{b}}{2} \\
\phi(t) & \text { for } & -\frac{T_{b}}{2}<t<\frac{T_{b}}{2} \\
\phi(t)+\phi\left(1,5 T_{b}\right)-\phi\left(2,5 T_{b}-t\right) & \text { for } & \frac{T_{b}}{2}<t<T_{b} \\
\frac{\pi}{2} & \text { for } & t>T_{b}
\end{array}\right.
$$

Following the above approximation there are only eight possible transmitted complex signals during the symbol period $\left[(n-0,5) T_{b},(n+0,5) T_{b}\right]$ (one set of eight complex signals for even $n$ and another set of eight for odd $n$ ), where $n$ is an arbitrary integer.

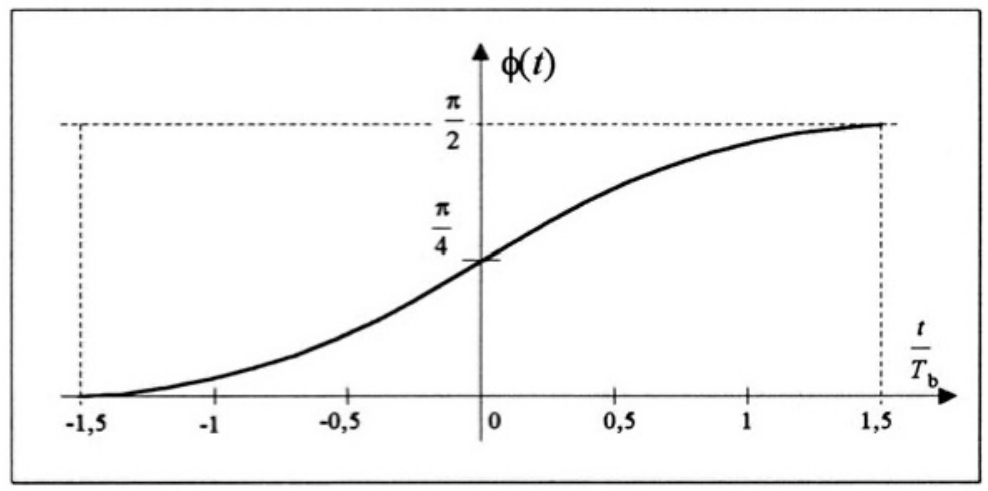

Figure l. Phase pulse-shaping function.

The state transition trellis diagram for the GSM modulated signals is shown in fig. 2. 


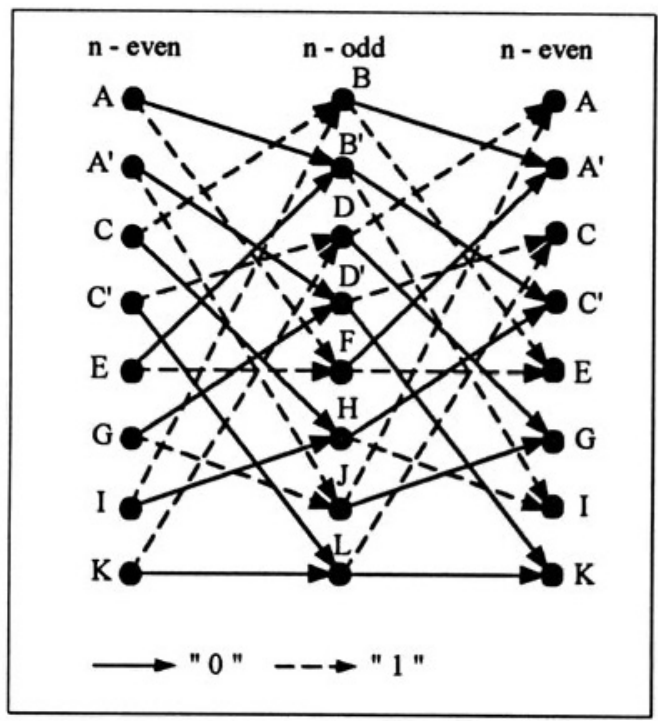

Figure 2. Trellis diagram of the GMSK modulated signal.

It should be noted that the trellis structure remains the same no matter what direction the transition comes from; from even to odd states or from odd to even states.

\section{GSM BURST STRUCTURE AND CHANNEL ESTIMATION}

The standard GSM burst structure is shown in fig. 3 .

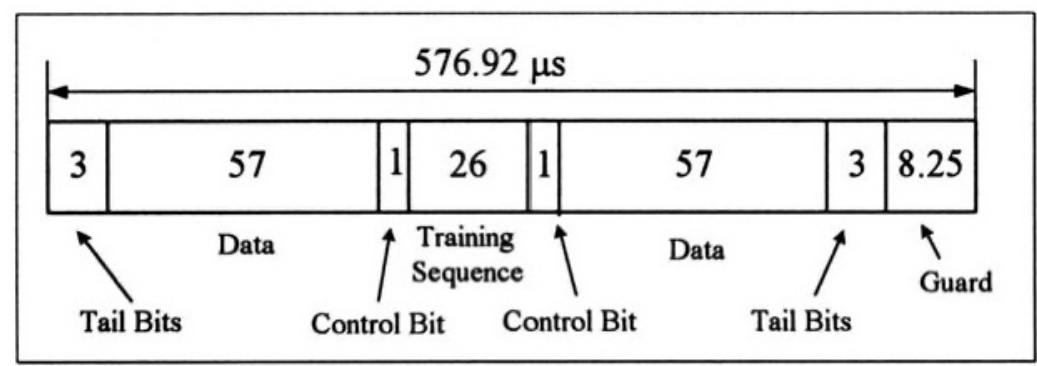

Figure 3. GSM burst structure.

The two 57 bits data fields are separated by two control flags and a 26 bit training sequence. This training sequence is used within GSM receiver for a precision synchronization and an estimation of channel impulse response. 
The estimate may be obtained for instance by correlating the received training sequence with a local copy held at the receiver.

Altogether eight such sequences have been defined within the GSM recommendations [3]. These sequences have been selected based on their good autocorrelation properties and their low cross-correlation properties between one another. Each sequence is composed of three distinct subsequences $\mathrm{X}, \mathrm{Y}$ and $\mathrm{Z}$, with sub-sequences $\mathrm{X}$ and $\mathrm{Z}$ being used twice within the entire sequence (fig. 4 [5]).

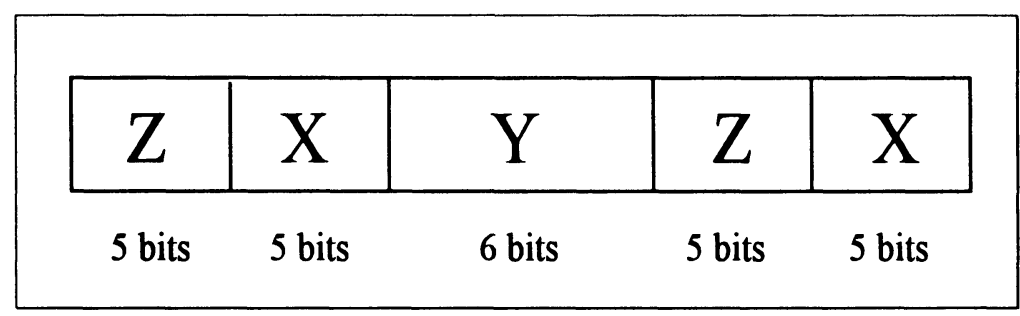

Figure 4. GSM training sequence structure.

The middle 16 bits in the entire 26 bits GSM training sequence allow the receiver to estimate the channel impulse response using five complex taps. It is crucial to note that all sequences share the central autocorrelation function peak surrounded by five „0" on each side. All possible sequences have been thoroughly analyzed. The autocorrelation results are portrayed in Fig. 5. Figure 5a presents the autocorrelation function of one of eight training sequences recommended by GSM, whereas figure $5 \mathrm{~b}$ presents the autocorrelation function of the improved training sequence calculated between the central 16 bits. 


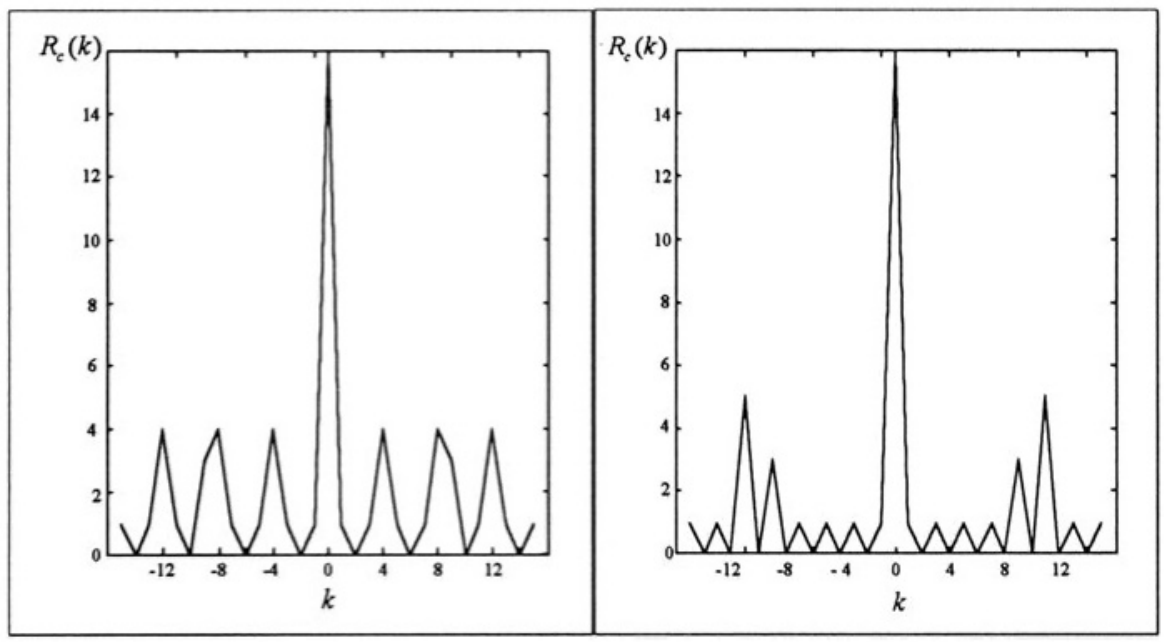

Figure 5a. Autocorrelation of one of the recommended training sequence used Figure $5 b$. Autocorrelation of the improved training sequence. in GSM system.

By comparing the two pictures, it can be observed that the improved autocorrelation function of training sequence is clearly better than that recommended by GSM. This can also be shown by simulation performance.

\section{GSM RADIO LINK SIMULATION}

The GSM simulation tool was designed according to ETSI (European Telecommunication Standard Institute) specifications [3]. The simulator has a flexible, modular structure in which each main GSM system element forms a basic simulation block. The simulator consists of: bits generator, channel encoder, interleaver, modulator GMSK, radio channel (with 3 propagation profiles TUx - Typical Urban, HTx - Hilly Terrain and RAx - Rural Area, where $x$ is the vehicle speed $[\mathrm{km} / \mathrm{h}][6])$, adaptive whitened matched filter, detector MLSE (with 8-state Viterbi algorithm), deinterleaver and channel decoder. Using the GSM simulator a number of packets containing the training sequence recommended by GSM and the improved version of training sequence were sent through a three channel models. The estimated BER performances for the TU50, HT100 and RA250 channel models are shown in fig. $6 \div$ fig. 8 .

In channels with a low dispersion of less than $5 \mu s$ (TU and RA profiles) a good performance for estimating the channel impulse response can be 
achieved by using both training sequences. However, the results are slightly better (about $0.1 \div 0.3 \mathrm{~dB}$ ) by using the new training sequence.

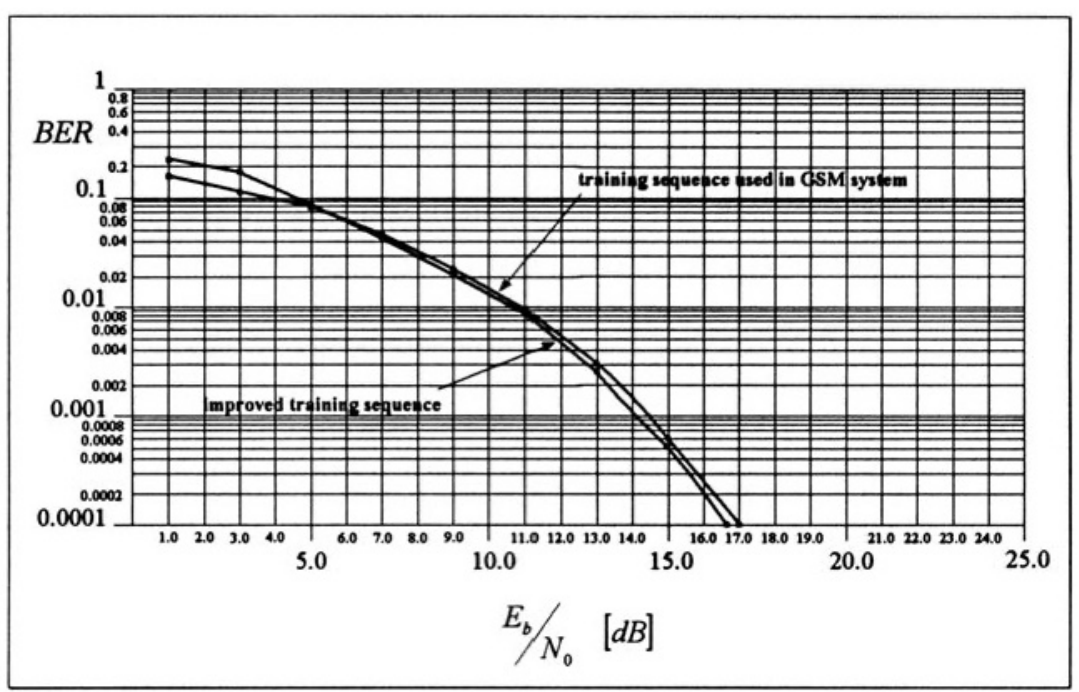

Figure 6. Simulation resuits under TU50.

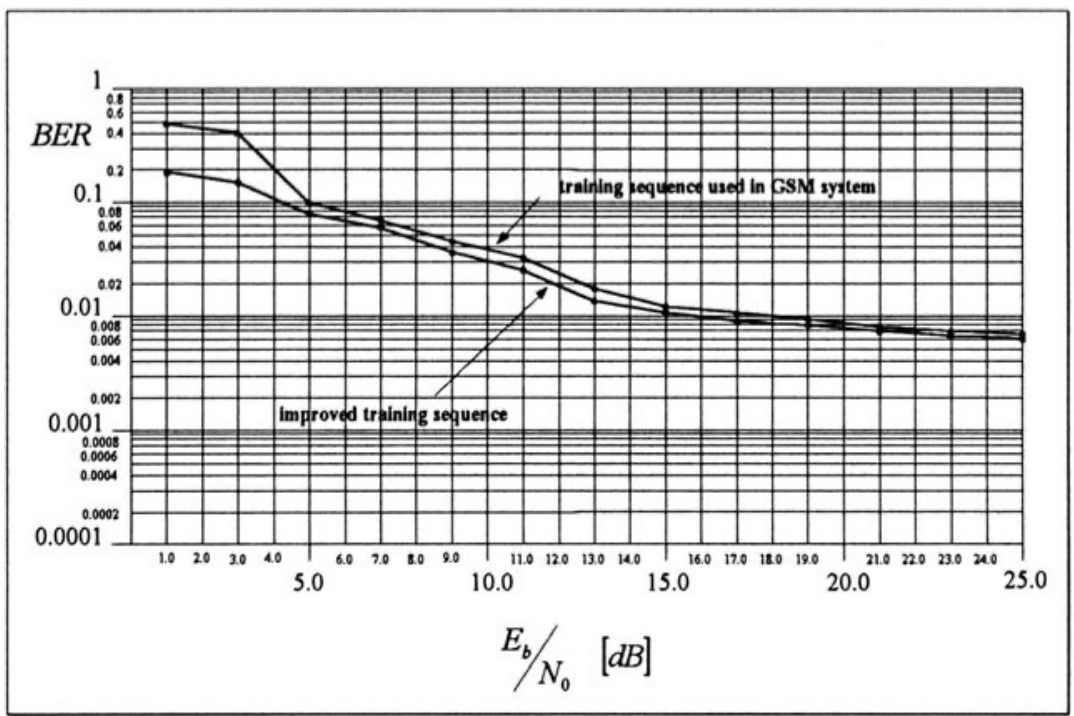

Figure 7. Simulation results under HT100. 


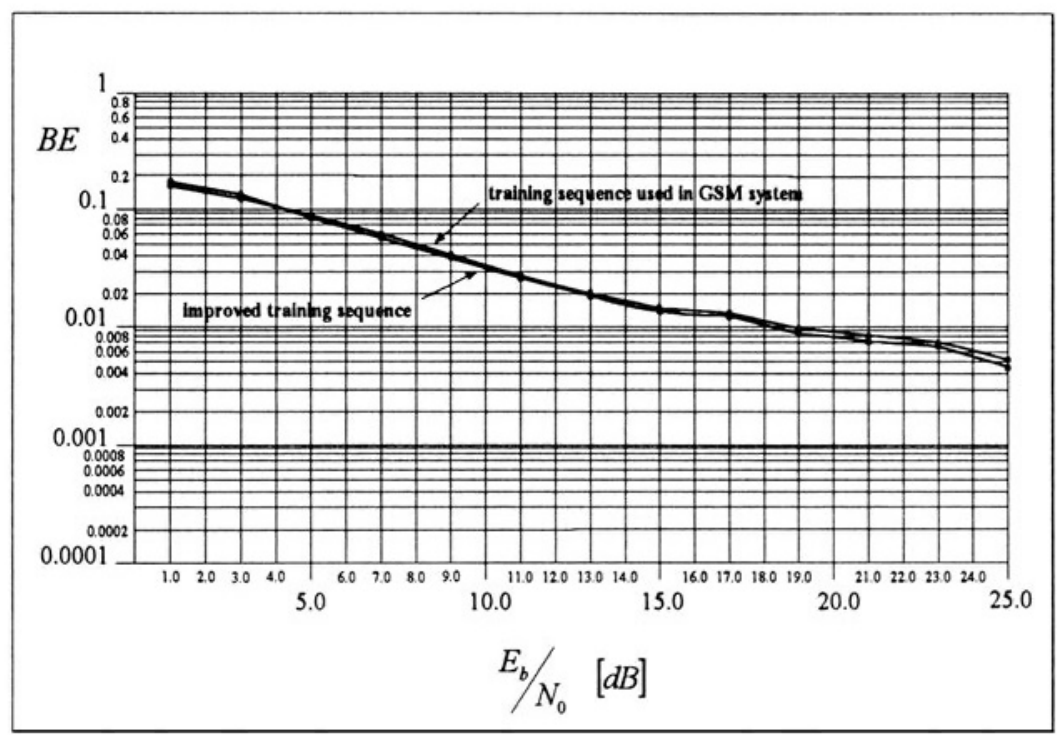

Figure 8. Simulation results under RA250.

In channels with a higher dispersion (HT profile), however, the results are clearly better by using the new training sequence (about ldB). This can by explained by the fact that the shape of the autocorrelation function of a new training sequence resembles the shape of the autocorrelation function of white noise (better estimation channel impulse response) [7].

\section{SUMMARY AND CONCLUSIONS}

The article evaluates two training sequences applied in digital cellular mobile communication systems for estimating the channel impulse response. It has been shown that a better sound quality may be obtained by using an improved version of training sequence than by using the training sequence recommended by GSM. Therefore, it can be argued that the training sequence used in GSM system has been not selected based on maximizing the quality of received signal through minimizing BER but rather on ensuring the maximum synchronization in the system. 


\section{REFERENCES}

[1] Forney G.: Maximum-Likelihood Sequence Estimation of Digital Sequences in the Presence of Intersymbol Interference. IEEE Transactions on Information Theory, vol. IT18, no. 3, pp. 363-378, May 1972.

[2] Murota K., Kenkichi H.: GMSK Modulation for Digital Mobile Radio Telephony. IEEE Transactions on Communications, vol. COM-29, no. 7, July 1981.

[3] GSM Technical Specifications. ETSI, Sophia Antipolis, 1992.

[4] Chen J. T., Paulraj A., Reddy U.: Multichannel Maximum-Likelihood Sequence Estimation (MLSE) Equalizer for GSM Using a Parametric Channel Model. IEEE Transactions on Communications, vol. COM-47, no. 1, pp. 53-63, January 1999.

[5] Joyce R. M., Ibbetson L. J., Lopes L. B.: Prediction of GSM Performance Using Measured Propagation Data. Proc. 46 ${ }^{\text {th }}$ Vehic. Technol. Conf., pp. 2246-2250, 1996.

[6] COST 207. Final Report, Digital Land Mobile Radio Communications. Commision of the European Communities, Luxemburg, 1989.

[7] Nahi N. E.: Estimation Theory and Applications. John Wiley \& Sons, 1969. 